\title{
Diversity Techniques in Wireless Body Area Network
}

\author{
Savita Sindhu ${ }^{\# 1}$, Dr.Shruti Vashist ${ }^{* 2}$ \\ \# Research Scholar, ECE, FET, Manav Rachna International University \\ Sector 43, SurajKund Road, Faridabad (Haryana, India) \\ ${ }^{1}$ savita.fet@mriu.edu.in \\ * Associate Professor, ECE, FET, Manav Rachna International University \\ Sector 43, SurajKund Road, Faridabad (Haryana, India) \\ ${ }^{2}$ shruti.fet@mriu.edu.in
}

\begin{abstract}
In WBAN various sensors and actuators are connected together either on the body, under the skin of person or on the clothes of the person. This network expands when the signals from the body are picked and sent over a wireless channel. The world that we live in has become very advanced, and hence it is required that the technology we use for communicating the signal should be very expeditious, i.e. our data carrying signals should profuse in excellently, so that data can be communicated without any intervention as the signals picked from body are of short range. But the data carrying signals that are relayed from one place to other often encounter various interferences in their path. One of the major problems in communicating wirelessly that has to be dealt with is fading. In WBAN if the signals can be captured with maximum information we can easily diagnose patient's disease for example; Cardiovascular diseases, diabetes control etc. Diversity is an important aspect that helps in dealing with the effects of fading. This paper focuses on the diversity techniques (Maximal Ratio Combining, Equal Gain combining, Selection Diversity) which are one of the popular mitigation methods; in different fading channels in WBAN. It also provides a comparative study of diversity techniques in fading channels w.r.t. to AWGN channel and three of the diversity techniques with respect to theoretical results.
\end{abstract}

Keyword- WBAN, Diversity, Fading, Fading channels, AWGN channel.

\section{INTRODUCTION}

Wireless communication has become an important constituent of the modern day world. Hence it becomes very important for any communication system to transfer data securely and reliably, especially when human being is considered. But the various elements present in the environment that surrounds the communication system often deteriorates the signal leading to drop in signal quality or loss of data, etc. The channels over which signals travel can be debilitated though numerous reasons, some of them being noise, loss in path, attenuation, fading.

It is very important that at the receiver end the received information should be same as what has been transmitted from the transmitter end. Buncha Sansoda, Somsak [15] proposed maximal ratio combining technique along with concatenated code(either RS or convolution codes) to improve the error performance of the system which was reduced due to multiple communication branching. The paper focused on the impact of Rician fading channel and BER with respect to Wireless body area network.[16] Proposed for on body propagation phenomena; body and multipath due to environment plays an important role in achieving relatively high diversity gain. Most of the on-body radio links shows low correlation of the diversity branches which implies that if power imbalance is taken care of then diversity plays very significant role. Channels either having non-line-of-sight condition or dynamic channels show maximum values of gain.

A. FADING

A signal when transferred from the transmitter side to the receiver often follows various propagation paths other than its normal route; this is because the signal gets reflected because of the interruptions present in its path, and each copy of the signal gets exposed to different levels of degradation and delay. Hence, signals obtained at the receiver become weak. This is known as fading [14].

Various types of fading are:

1) Flat fading

2) Frequency selective fading

3) Fast fading

4) Slow fading

Fading has an adverse effect on the quality of the signal received. Therefore it becomes important to eliminate the effects of fading. [2-3] 
This paper is organized as follows: Section 2 presents various fading channels and the effect of diversity techniques on signal. Section III covers the effect of diversity combining techniques on the signal .Section IV gives the analysis of the result based on comparison of various combining techniques on the signal with respect to AWGN channel ;comparison of EGC,MRC,SC diversity techniques and finally in section V conclusions are drawn.

\section{FADING CHANNELS AND DIVERSITY TECHNIQUES}

In wireless Fading channels is a channel over which fading occurs [5].

\section{A. RAYLEIGH FADING CHANNEL}

Rayleigh fading is used when the direct line of sight is not present, and the signal is distracted before arriving at the receiver. Here, speed of the channel is determined by the speed of the moving transmitter or receiver. The model that is used in such type of a scenario is known as Rayleigh fading model.

When line of sight is not present the signal that is received is represented as [6]:

$$
s(t)=\sum_{i=1}^{N} \mathrm{a} \cos \left(\mathrm{W}_{c} \mathrm{t}+\emptyset_{i}\right)
$$

B. RICIAN FADING CHANNEL

Rician fading is used when a line of sight is present. Under such environment Rician distribution is used.

The received signal through rician fading channel is represented as [6]:

$$
\mathrm{s}(\mathrm{t})=\sum_{i-1}^{N-1} a_{i} \cos \left(w_{c} t+w_{d i} t+\emptyset_{i}\right)+k_{d} \cos \left(w_{c}+w_{c} t\right)
$$

where the constant $k_{d}$ is the strength of the direct component, $\omega_{\mathrm{d}}$ is the Doppler shift along the line-of-sight path, and $w_{d i}$ are the Doppler shifts along the indirect paths. The envelope in this case has a Rician density function given by $[4,11]$ :

$$
\mathrm{f}(\mathrm{r})=\frac{r}{\sigma^{2}} e^{\left\{\frac{r^{2}+k_{d}^{2}}{2 \sigma^{2}}\right\} \cdot I_{0}\left\{\frac{r k_{d}}{\sigma^{2}}\right\}}
$$

where $I_{0}(0)$ the $0^{\text {th }}$ order is modified Bessel function of the first kind

\section{DIVERSITY}

Diversity is a technique which is used to remove fading in wireless communication. In diversity, the basic ideology is to transmit the signal using more than one path [1][10]. The reason behind transmitting the signal, through more than one path is that suppose in one path signal undergoes a high level of fading then there might be a probability that by travelling through other path the same signal experiences less amount of fading.

Types of diversity techniques [8]:

\section{1) Antenna diversity}

Antenna diversity can be employed either at the transmitter side or the receiver side. In antenna diversity more than one antenna are used to transmit or receive the signals, and the signal having the best power will be selected.

\section{2) Frequency diversity}

In frequency diversity the signals received at the receiver are carried by more than one frequency band, as different frequency bands will encounter different amount of fading.

3) Time diversity

In time diversity different time slots are used to transfer the signal. Here each signal will be transmitted at different time intervals, to combat fading.

\section{4) Polarization diversity}

Here the antenna used has dual polarization which is orthogonal to each other. The signals sent by the dual polarized antenna are not correlated with each other because the polarizations is orthogonal to each other.

\section{DIVERSITY COMBINING}

In order to get the full advantage from the diversity scheme, combining of the diversity should be done at the receiver. [10] In diversity combining the signals obtained from the different branches are combined to get a single powerful signal. When the signals are combined it helps in improving system performance. There is various diversity combining techniques that are used to increase the performance of the system [4].

\section{1) Maximal Ratio Combining}

In this ombining technique signals obtained from various diversity branches are first weighted and co phased before they are combined. The branches having stronger signals are amplified and the weak signal branches are removed. Finally they are combined and the resultant output is obtained. In Maximal Ratio Combining all the branch information is used. [7][9] 


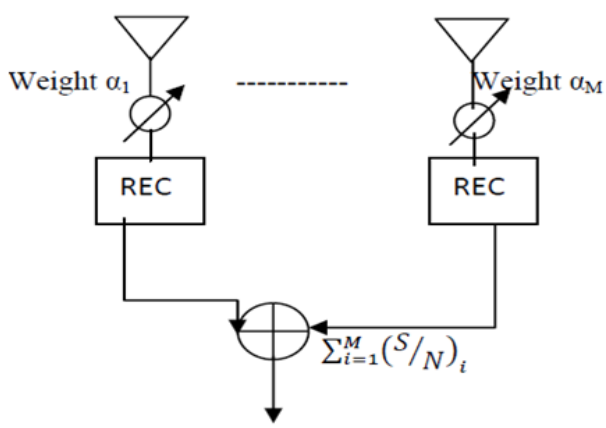

Fig1. Maximal Ratio Combining

\section{2) Equal Gain Combining}

In Equal Gain combining the weights of each and every branch is determined beforehand and they are not changed. Then the signals are co-phased and added, and the output is connected to the demodulator. [13]

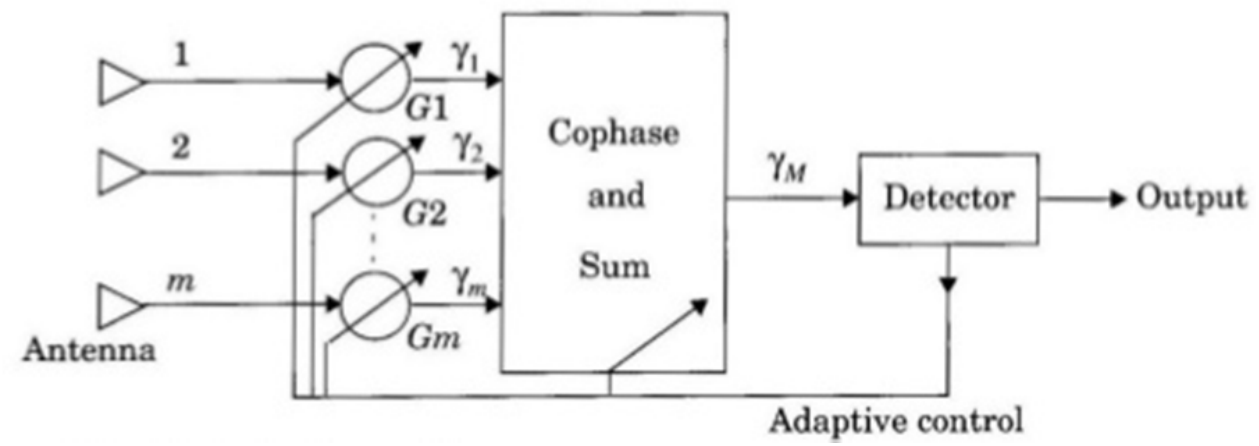

Fig2. Equal Gain Combining

\section{3) Selection Combining}

Selection combining follows a simple procedure. It selects the signal that is the best which means it has the highest signal to noise ratio from the available signal copies.[7]

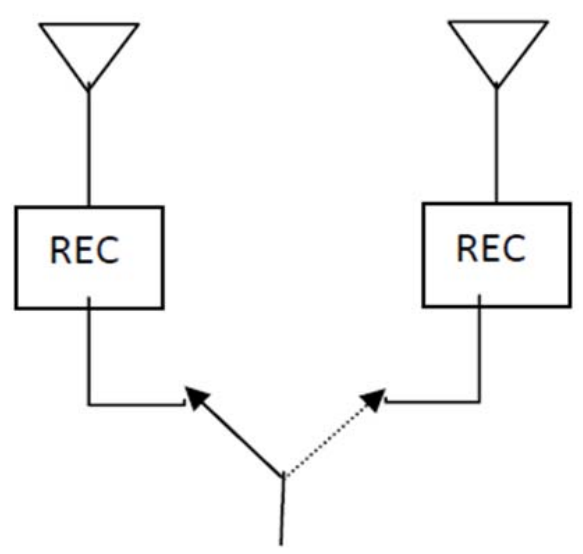

Fig3. Selection combining

\section{RESULTS AND DISCUSSION}

Using the combining techniques the SNR versus graph has been calculated in Rayleigh and Rician channel with the help of MATLAB software[5,11-12].Figure 4 given below shows theoritical and simulated results of maximal rati combining techniques in rayleigh channel.

It can be observed that the results calculated using simulation method is better as compared to that of theortical one which can be observed in Table 1 . 


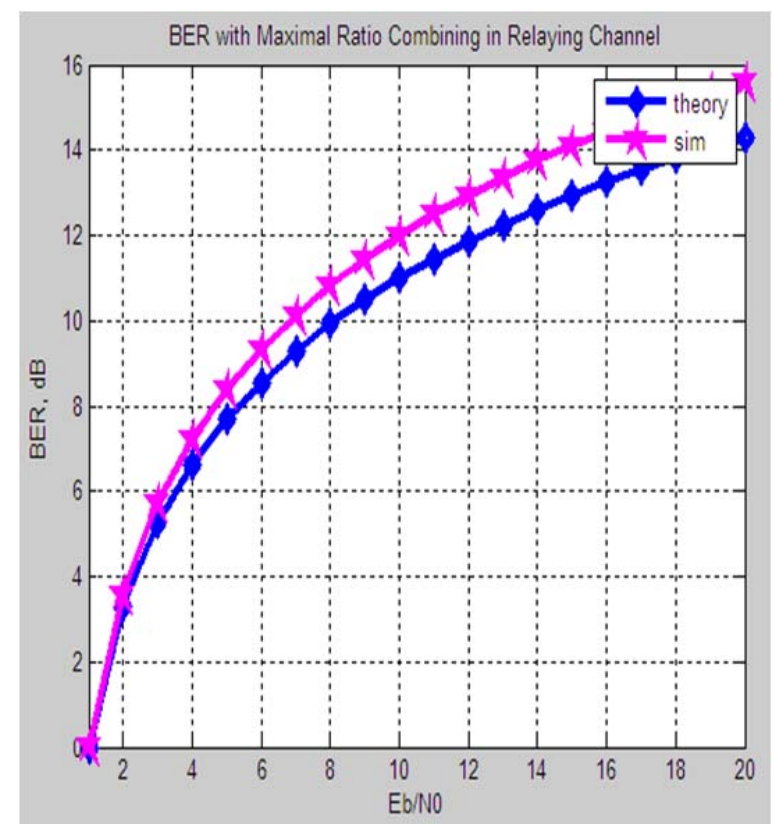

Fig 4.Maximal ratio Combining in Rayleigh Channel

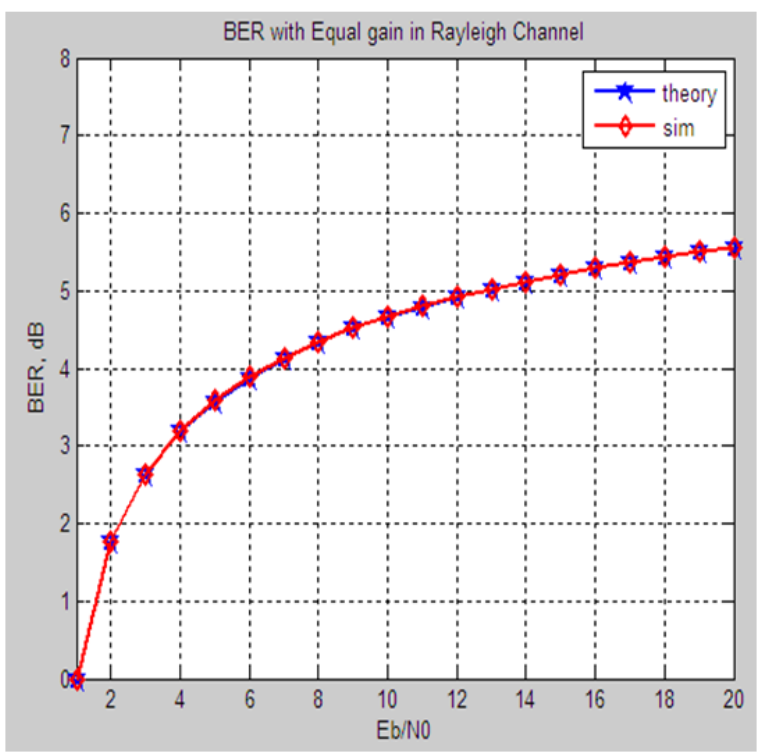

Fig 5.Equal Gain Combining in Rayleigh Channel

This figure shows theoretical and simulation results of Equal Gain Combining in Rayleigh Channel. In this diversity technique the results of theoretically calculated values and simulated values at most. 


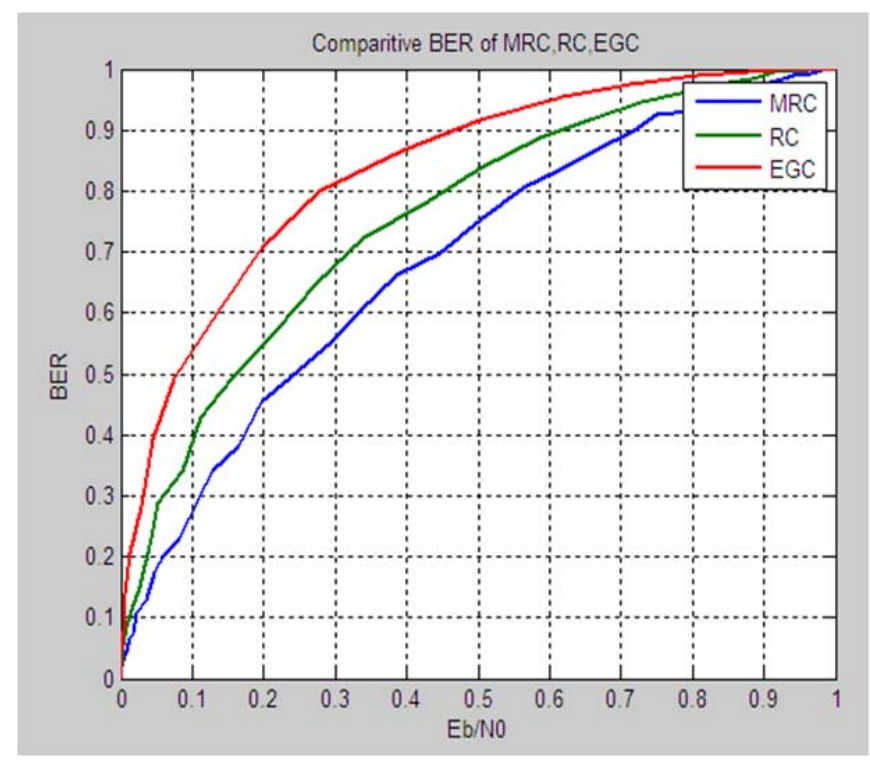

Fig 6. Comparison between Maximal ratio combining and Equal gain combining in Rayleigh channel.

Table 1: BER of Rayleigh channel, EGC and MRC diversity techniques

\begin{tabular}{|c|c|c|c|}
\hline $\mathrm{E}_{\mathrm{B} /} \mathrm{N}_{\mathrm{O}}$ & BER of EGC & BER of RC & BER of MRC \\
\hline 0.1 & 0.27 & 0.37 & 0.55 \\
\hline 0.2 & 0.43 & 0.55 & 0.72 \\
\hline 0.3 & 0.56 & 0.69 & 0.82 \\
\hline 0.4 & 0.68 & 0.76 & 0.87 \\
\hline 0.5 & 0.74 & 0.83 & 0.92 \\
\hline 0.6 & 0.83 & 0.90 & 0.95 \\
\hline 0.7 & 0.89 & 0.96 & 0.96 \\
\hline 0.8 & 0.94 & 0.98 & 0.98 \\
\hline 0.9 & 0.97 & 0.98 & 0.99 \\
\hline
\end{tabular}

It can be observed that the Maximum Ratio Combining performance is the best as compared to Equal Gain Combining and Selection Combining in Rayleigh fading channel. For the same SNRs signals applied to both branches, the output of maximal ratio combiner is more than equal gain combiner. This is due to ability of updating the weight coefficient in Maximal Ratio combining technique.

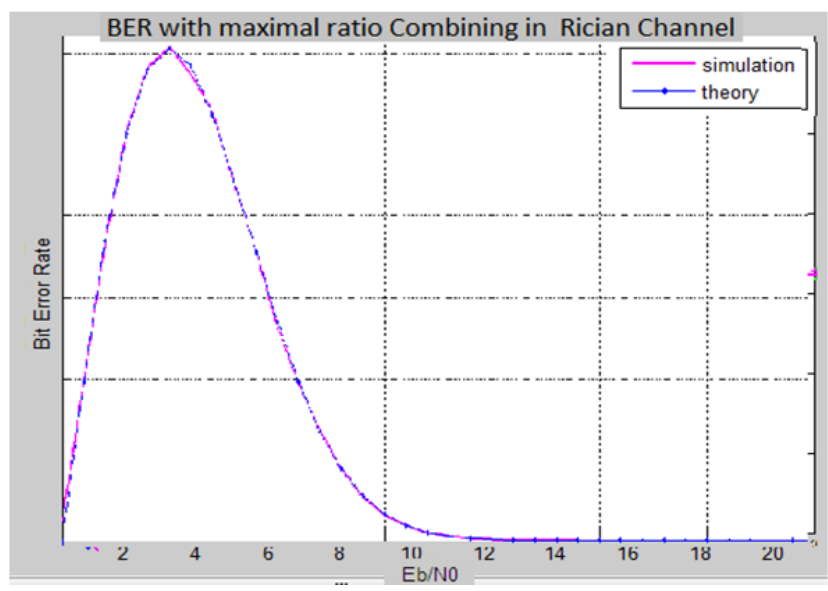

Fig 7. Maximal Ratio Combining In Rician channel 


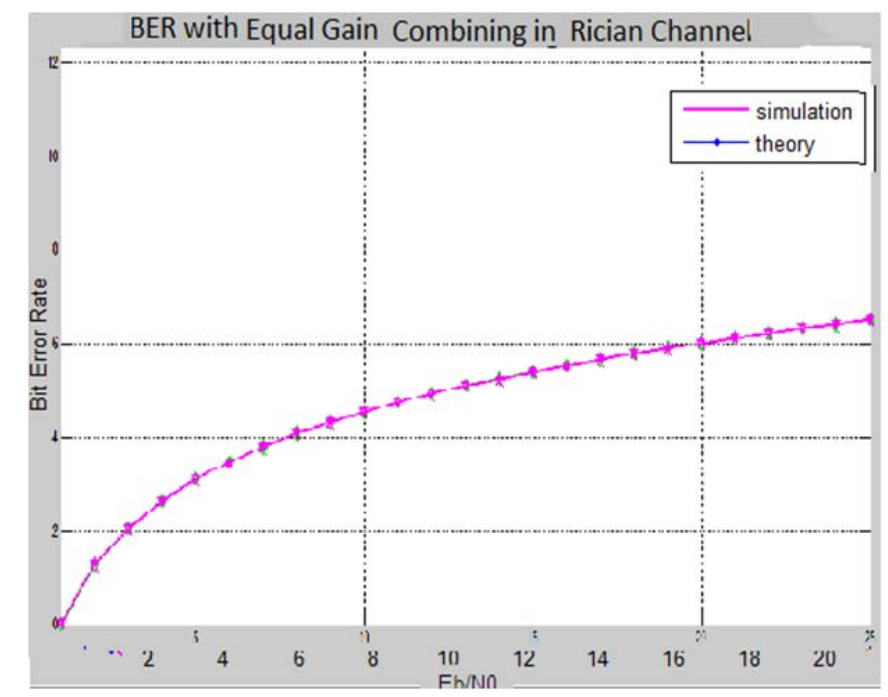

Fig 8. Equal Gain Combining in Rician channel.

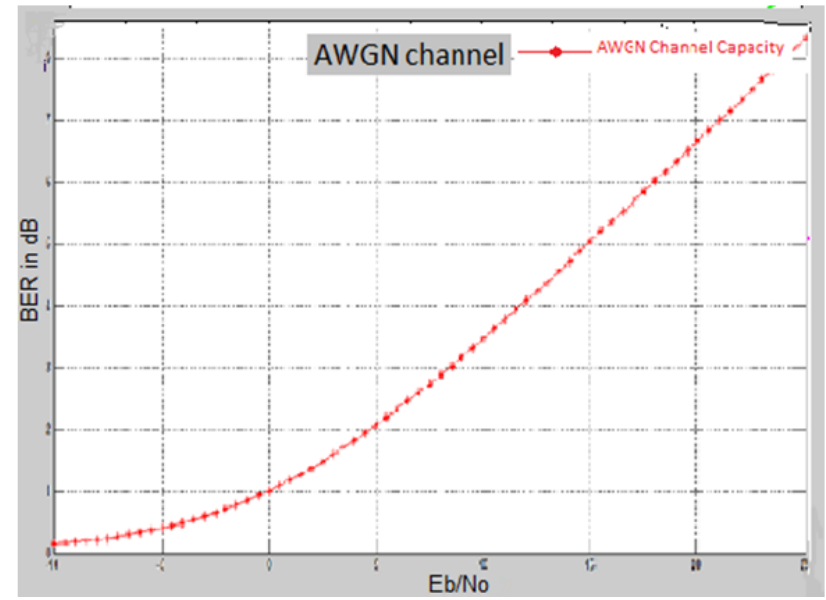

Fig 9. Ideal case of AWGN channel.

This figure shows AWGN channel which is an ideal case in WBAN fading as it is considered as reference value which can be clearly observed from the table 2 which shows that Maximal Ratio Technique in Rayleigh and Rician technique shows better results.

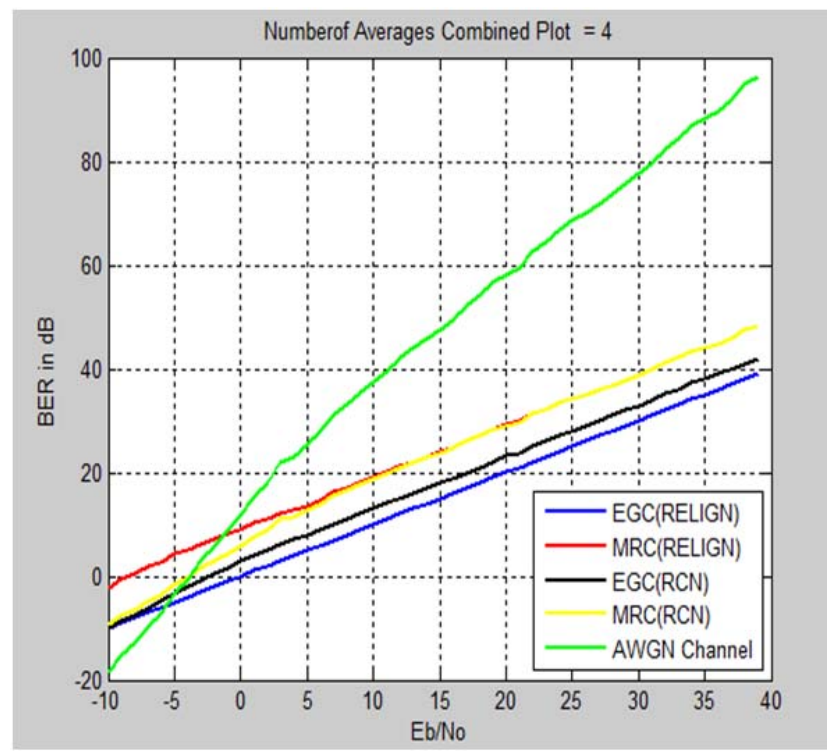

Fig 10. Comparison of Diversity techniques in different fading channels with respect to AWGN channel. 
Table 2: Comparison of Diversity techniques in different fading channels with respect to AWGN channel.

\begin{tabular}{|l|l|l|l|l|l|}
\hline Eb/No & AWGN channel & \multicolumn{2}{l|}{ Rayleigh channel } & \multicolumn{2}{l|}{ Rician channel } \\
\hline & & MRC & ECG & MRC & ECG \\
\hline 0 & 11 & 8 & 2 & 6 & 0 \\
\hline 5 & 21 & 12 & 5 & 11 & 3 \\
\hline 10 & 30 & 16 & 10 & 15 & 8 \\
\hline 15 & 38 & 20 & 13 & 19 & 11 \\
\hline 20 & 47 & 24 & 18 & 23 & 16 \\
\hline 25 & 54 & 27 & 21 & 26 & 19 \\
\hline 30 & 59 & 30 & 23 & 29.5 & 21 \\
\hline
\end{tabular}

Above table shows the BER for BPSK modulation schemes with Equal Gain combining and Maximal ratio combining technique in Rayleigh and Rician fading channels. It can be concluded that BER for BPSK with single antenna at receiving side is same in both Maximal Ratio Combining technique and Equal Gain Combining technique. But when two or more receiving antennas are used; Maximal Ratio Combining technique performs better than Equal Gain Combining technique with BPSK.

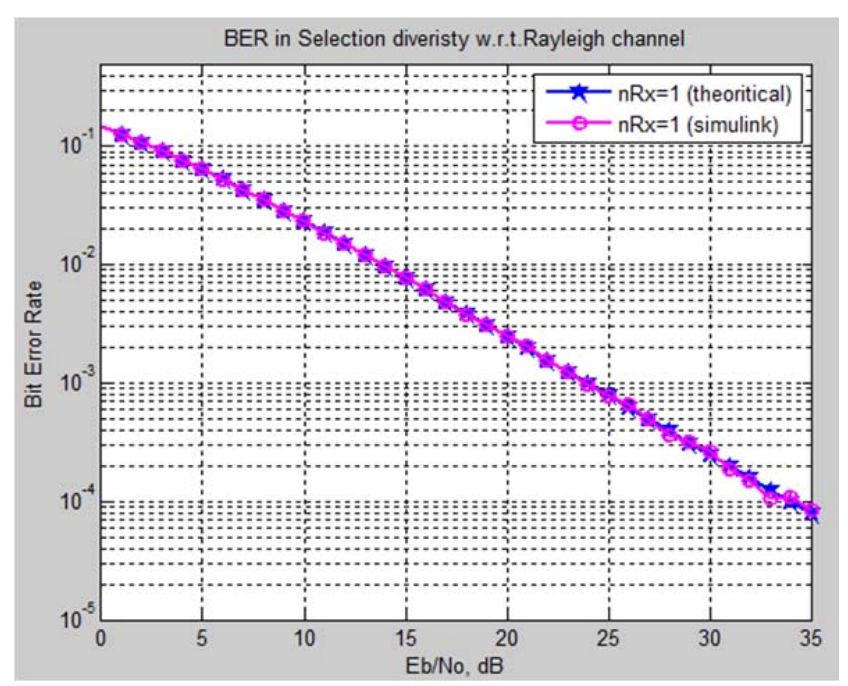

Fig 11. Selection Diversity in Rayleigh channel

Figure 11 shows selection diversity in Rayleigh channel theoretical and using simulink .Is shows almost equal results when compared.

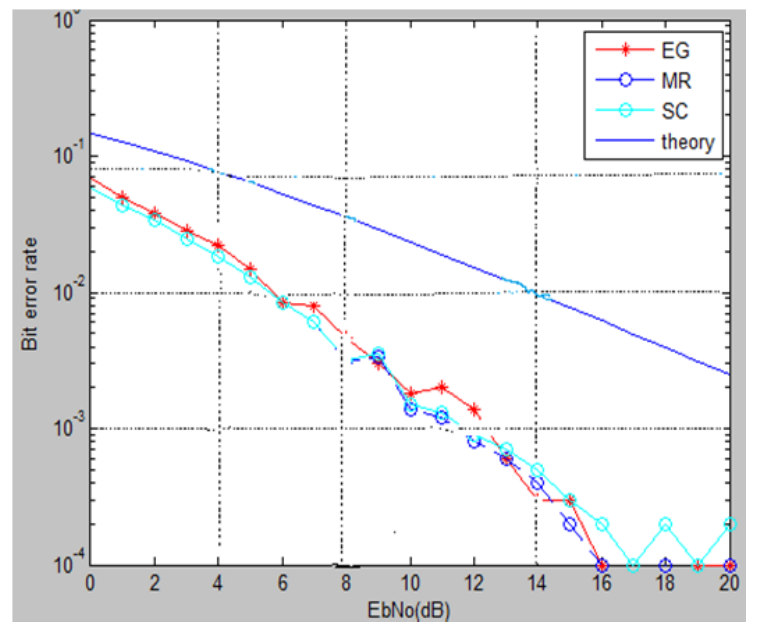

Fig 12. Comparison of Diversity techniques 
Table3:Comparative study of three diversity techniques:

\begin{tabular}{|l|l|l|l|}
\hline $\mathrm{Eb} / \mathrm{No}$ & Selection diversity & EGC & MRC \\
\hline 0 & 0.0684 & 0.0576 & 0.0574 \\
\hline 4 & 0.0500 & 0.0431 & 0.0431 \\
\hline 8 & 0.0377 & 0.0340 & 0.0337 \\
\hline 12 & 0.0284 & 0.0246 & 0.0240 \\
\hline 16 & 0.0223 & 0.0183 & 0.0180 \\
\hline 18 & 0.0146 & 0.0128 & 0.0121 \\
\hline 20 & 0.0083 & 0.0083 & 0.0081 \\
\hline
\end{tabular}

The performance of EGC is superior to selection diversity, however, marginally inferior to MRC, which can be seen from above table.

\section{CONCLUSION AND SCOPE FOR FUTURE WORK}

The above paper presented one of the mitigation methods (Fig 10) i.e. Diversity technique which has been applied on two different fading channels and is compared with reference to AWGN channel. Two different channels used were Rayleigh and Rican fading channel which were defined as there was no dominant line of sight. When signals from WBAN were captured, and then the chances of fading increased. There may or may not be a dominant line of sight. Diversity combining has been applied which has been one of the essential techniques which helped in reducing the effects of fading. In this paper selection diversity results are shown, Maximal Ratio Combining and Equal Gain Combining in Rayleigh fading channel and Rician fading channel has been compared with respect to AWGN channel. The result showed that AWGN channel outperforms the other two fading channels. Maximal ratio combining technique in Rayleigh channel performs next to better or worse AWGN channel. Additionally comparative study of three of the diversity techniques presented here was also shown w.r.t. figure 12. In the $2^{\text {nd }}$ comparative study table also Maximal ratio combining technique performs better than other two techniques. Further viterbi decoder or equalization techniques can be used in future for fading mitigation as the ultimate goal is to receive signal with maximum information and less distortion.

\section{REFERENCES}

[1] S. M. Metev and V. P. Veiko, Laser Assisted Microtechnology, 2nd ed., R. M. Osgood, Jr., Ed. Berlin, Germany: Springer-Verlag, 1998.

[2] J. Breckling, Ed., The Analysis of Directional Time Series: Applications to Wind Speed and Direction, ser. Lecture Notes in Statistics. Berlin, Germany: Springer, 1989, vol. 61.

[3] S. Zhang, C. Zhu, J. K. O. Sin, and P. K. T. Mok, “A novel ultrathin elevated channel low-temperature poly-Si TFT," IEEE Electron Device Lett., vol. 20, pp. 569-571, Nov. 1999.

[4] M. Wegmuller, J. P. von der Weid, P. Oberson, and N. Gisin, "High resolution fiber distributed measurements with coherent OFDR," in Proc. ECOC'00, 2000, paper 11.3.4, p. 109.

[5] R. E. Sorace, V. S. Reinhardt, and S. A. Vaughn, "High-speed digital-to-RF converter,” U.S. Patent 5668 842, Sep. 16, 1997.

[6] (2007) The IEEE website. [Online]. Available: http://www.ieee.org/

[7] M. Shell. (2007) IEEEtran webpage on CTAN. [Online]. Available: http://www.ctan.org/tex-archive/macros/latex/contrib/IEEEtran/

[8] FLEXChip Signal Processor (MC68175/D), Motorola, 1996.

[9] “PDCA12-70 data sheet," Opto Speed SA, Mezzovico, Switzerland.

[10] A. Karnik, "Performance of TCP congestion control with rate feedback: TCP/ABR and rate adaptive TCP/IP," M. Eng. thesis, Indian Institute of Science, Bangalore, India, Jan. 1999.

[11] J. Padhye, V. Firoiu, and D. Towsley, "A stochastic model of TCP Reno congestion avoidance and control," Univ. of Massachusetts, Amherst, MA, CMPSCI Tech. Rep. 99-02, 1999.

[12] Wireless LAN Medium Access Control (MAC) and Physical Layer (PHY) Specification, IEEE Std. 802.11, 1997.

[13] [13] Shruti Sridharan, "Diversity: A Fading Mitigation Technique" ,International Journal of Advanced Research in Electronics and CommunicationEngineering, Vol - 3, Issue 8, year-August 2014.

[14] Theodore S. Rappaport, "Wireless Communications: Principles and Practice", Prentice Hall, year-2002

[15] Buncha Sansoda ; Somsak Choomchuay "Performance evaluation of error correction code in MIMO scheme for WBAN channel", International Symposium on Intelligent Signal Processing and Communication Systems (ISPACS), 2014 IEEE

[16] A. A. Serra A. Guraliuc P. Nepa G. Manara P. S. Hall \&quot;Diversity gain measurements for body-centric communication systems\&quot; Int. J. Microw. Opt.Technol.,vol.3no.3,pp.283-289Jul.2008

\section{AUTHOR PROFILE}

Savita Sindhu is a Research Scholar and doing her research work in the area of Wireless communication and Wireless Body Area Network (WBAN). She has been associated with teaching from the past 9 years. Currently she is working with Manav Rachna International University Faridabad as an Assistant Professor in Electronics \& Communication Engineering Deptt. She had published more than 12 papers in esteemed National, International journals and conferences. She also guided 4 M.Tech. students till date.

Dr.Shruti Vashist has 18 yrs of Teaching (U.G., P.G.) experience in the Manav Rachna International University ,Faridabad ,India as Professor. Obtained B.E. (Electronics Engineering) degree from College of Engineering , Chandrapur ,Nagpur University in 1993 ,M.Tech(Electronics and Communication) from Rajasthan University in 2006 and completed Ph.D from Manav RachnaInternational university in 2015.Area of interest is Wireless and Mobile communication, Analog Electronics and Microstrip Antennas. Published three books and many papers in National and international Journals and conferences .Has been actively participating on behalf of ECE department in various projects and consultancies. 